\title{
A Novel Approach to Make Decision in Intelligent Decision Support systems

\author{
Yehong Han
}

Institute of Information Science and Engineering, Qilu Normal University, Jinan, 250014, China

Email: sdzzhyh@163.com

Keywords: Rough sets; Intelligent decision support system; Uncertainty reasoning; Artificial intelligence

\begin{abstract}
Although decision ability of intelligent decision support systems has been greatly improved, the method of decision making under uncertainty is a difficult point. In this paper, an algorithm based on rough set theory is introduced, and the approach to make decision in intelligent decision support systems is emphasized. In order to make decision, the minimum set of decision rules should be computed by simplification of decision table firstly, and the result of decision can be obtained by comparing the new object with the minimum set of decision rules and computing the weight of every conclusion in the new object's matching set. Both subjective factors representing the users' preferences for various attributes and objective factors such as support degree of decision rules are taken into account in the process of reasoning, and the proportion of the subjective factors and the objective factors can be adjusted by the ratio of subjective factors. The approach can be used to make decision under certain and uncertain environment, and can better reflect the needs of users.
\end{abstract}

\section{Introduction}

Intelligent decision support system (IDSS) [1][2][3] which can be used to assist decision maker in solving the complex decision problems by logical reasoning is the product of the decision support system (DSS) combined with artificial intelligence technology. But decision making based on the uncertainty or incomplete knowledge is a difficult point in IDSS.

In order to solve above question, a method based on rough sets theory[4][5] is given in the paper. The model of IDSS based on rough sets theory is created as shown in Fig .1. The initial database in IDSS can be described as knowledge representation systems or decision tables in this model, and the inference machine is a minimum set of decision rules obtaining by simplification of decision table. The result of decision can be obtained by comparing the new object with the minimum set of decision rules and computing the weight of every conclusion in the new object's matching set. In order to make the reasoning result meet the requirement of users, a novel approach considering subjective factors of conditions of problem requests to make decision is proposed and elaborated. In this method, both the subjective factors such as the user's interests and the objective factors such as the minimum set of decision rules are synthetically reflected in the process of reasoning, and the proportion of the subjective factors and the objective factors can be adjusted according to the actual needs of the users. Consequently the new approach is more suitable for making decision in intelligent decision support systems.

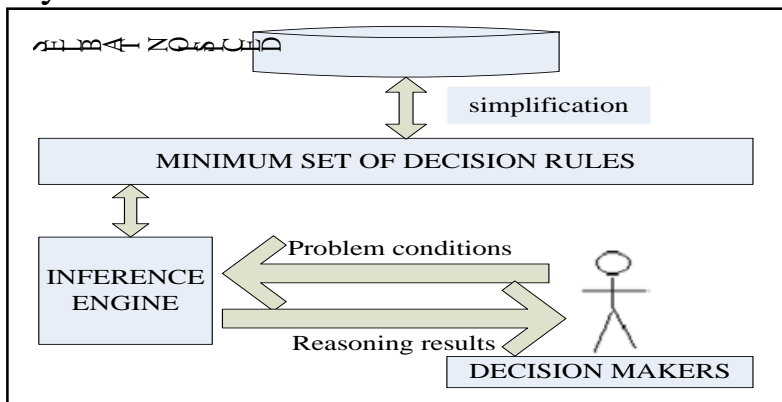

Fig. 1. The model of IDSS based on rough sets 


\section{Notations and Definitions}

In order to present the method to make decision in IDSS based on rough sets theory, notations and definitions that are germane to the paper will be introduced.

Knowledge representation system [6]can be defined a pair $K=<U, A>$, where $U$ is a nonempty and finite set, and $A$ is a nonempty and finite set of primitive attributes. Decision table [7]referring to knowledge representation system can be denoted $T=<U, A, C, D>$, where $C, D \subset A$ are called condition and decision attributes respectively. Decision rule which can be denoted by $r$ is a implication $\alpha \rightarrow \beta$, where $\alpha$ and $\beta$ are referred to as the predecessor and the successor of $\alpha \rightarrow \beta$ respectively. $\alpha, \beta$ can be described as the formula of $\left(a_{1}, v_{1}\right) \wedge\left(a_{2}, v_{2}\right) \wedge \ldots \wedge\left(a_{n}, v_{n}\right)$, where $a_{i}$ $(i=1,2, \ldots, n)$ is a attribute of a decision table and $v_{i} \quad(i=1,2, \ldots, n)$ is the value of the attribute $a_{i}$.

The minimum set of decision rules[8]which consists of the minimization of decision rules can be got by simplification of decision table. The minimum set of decision rules can be denoted $M$.

A new object which will be denoted $\mathrm{x}$ can be seen as a predecessor of a decision rule. The set of condition attributes in the new object $\mathrm{x}$ can be defined all attributes of the new object $\mathrm{x}$ and will be denoted $S_{-} C A(x)$. Condition attributes set of the decision rule $\mathrm{r}$ is all condition attributes of a decision rule $\mathrm{r}$ and will be denoted $S_{-} C A(r)$.

Let $T=<U, A, C, D>$ be a decision table(the number of elements in $C$ is $n$ and the number of elements in $D$ is $m$ ) , a sample of the decision table $T$ can be represented as $\left(a_{1}, v_{1}\right) \wedge\left(a_{2}, v_{2}\right) \wedge \ldots \wedge\left(a_{n}, v_{n}\right) \rightarrow\left(a_{n+1}, v_{n+1}\right) \wedge\left(a_{n+2}, v_{n+2}\right) \wedge \ldots \wedge\left(a_{n+m}, v_{n+m}\right)$, where $a_{i} \in C \quad(i=1,2, \ldots, n), a_{n+i} \in D$ $(i=1,2, \ldots, m)$, and $v_{i} \quad(i=1,2, \ldots, n+m)$ is the value of the attribute $a_{i}$. Let ${ }^{r}: \alpha \rightarrow \beta$ be a decision rule, if $\left(a_{1}, v_{1}\right) \wedge\left(a_{2}, v_{2}\right) \wedge \ldots \wedge\left(a_{n}, v_{n}\right) \rightarrow \alpha$, the row of the decision table supports the decision rule $r$. In a decision table, the number of samples which support the decision rule $r$ will be called the degree of support of decision rule and will be denoted sup port $(r)$.

Decision rules are obtained by means of simplification of a decision table based on rough set theories, and the set of decision rules will be denoted $R U L$. Let $\mathrm{x}$ be a new object, and $\alpha \rightarrow \beta$ be a decision rule satisfying ${ }^{x \rightarrow \alpha}$, the rule $\alpha \rightarrow \beta$ will be called that the rule matches the new object. the matching set of the new object ${ }^{X}$ consists of all decision rules which match the new object ${ }^{X}$, will be denoted $R U L(x)$.

Proposition 1 Three case as follows will be inferred by matching the new object with every rules of the set of decision rules:

(1) The new object matches a rule or several rules and values of decision attributes are same.

(2) The new object matches several rules and values of decision attributes are not same.

(3) The new object does not match any decision rules.

\section{A Novel Approach to Make Decision Considering Subjective Factors}

Only uncertainty reasoning which have been classified in Proposition 1 will be introduced in the paper. Because the importance of each attribute is given according to subjective factors such as needing and fancy of users, a method of uncertainty reasoning considering subjective factors will be presented in the following

(1)The new object matches several rules whose decision attributes' values are not same

Definition1 The degree of significance with every attribute ${ }^{a}$ of the new object which will be denoted $\operatorname{sig}(a)$, is a real number satisfying $0 \leq \operatorname{sig}(a) \leq 1$ and $\sum_{a \in S_{-C A(x)}} \operatorname{sig}(a)=1$. It reflects the degree of user's preference for different attributes.

Definition2 The fit degree of every conclusion in the matching set of the new object ${ }^{x}$ will be denoted fitness $(x, \beta)$, and can be computed by formula (1)

$$
\text { fitness }(x, \beta)=\bigcup_{\alpha \rightarrow \beta \in R U L(x)} \sum_{a \in S_{-} C A(\alpha \rightarrow \beta)} \operatorname{sig}(a)
$$

Definition3 The Standardized fit degree of every conclusion in the matching set of the new object ${ }^{x}$ can be obtained by formula (2), and will be denoted $s$ tandard fitness $(x, \beta)$; 
$s \tan \operatorname{dard}_{-}$fitness $(x, \beta)=\frac{\operatorname{fitness}(x, \beta)}{\sum_{\beta \in \operatorname{decision}(x)} \operatorname{fitness}(x . \beta)}$

decision $(x)=\{\beta \mid \alpha \rightarrow \beta \in R U L(x)\} \quad$. there are two restrictive conditions in the process of reasoning: $S_{-} C A(x) \supseteq \bigcup_{r \in R U L} S_{-} C A(r)$ and $0<\operatorname{sig}(a)<1$. The two restrictive conditions can imply $\sum_{\beta \in \operatorname{decision}(x)} f i t n e s s(x . \beta) \neq 0$, and both illustrate that all attributes of the new object are useful and can't be ignored in the process of reasoning.

Definition4 The general weight will be denoted weight $(x, \beta)$ and can be computed by formula (3): weight $(x, \beta)=\theta \times$ standard_fitness $(x, \beta)+(1-\theta) \times$ reliability $(x, \beta)$

The value of reliability $(x, \beta)$ which is the reliability of the conclusion $\beta$ is computed by plenty of historical data. The value of reliability $(x, \beta)$ reflects objective factors of reasoning, so the reliability of the conclusion $\beta$ can be called objective weight of conclusion $\beta$. The value of $\theta$ will be called the ratio of subjective factor, and $0 \leq \theta \leq 1$.

Proposition 2 The following property is obvious: $0 \leq$ weight $(x, \beta) \leq 1$ and $\sum_{\beta \in \operatorname{decision}(x)} \operatorname{weight}(x, \beta)=1$

When the new object matches several rules and values of decision attributes are not same, the algorithm of decision making considering subjective factors can be described as follows:

Input: a minimum set of decision rules $M$; a new object $x$;

Output: a result of the decision;

Step1: Allot a degree of significance with every attribute $a$ of the new object.

Step2: Computer the fit degree of every conclusion in the matching set of the new object $x$ by formula (1);

Step3: Standardize fit degrees of all the conclusions in the matching set of the new object $x$ by formula (2)

Step4: Compute the general weight by formula (3)

Step5: Select the conclusion whose value of general weight is the maximum as the final result of uncertain reasoning.

In this algorithm, the value of $\theta$ can be used to adjust the proportion of subjective factors and the objective factors. When the value of $\theta$ is bigger, subjective factors is attached more importance by decision makers. When $\theta=0$, only objective factors are considered, and the algorithm of uncertain reasoning turns to traditional vote.

\section{(2)The new object does not match any decision rules}

Definition5 The matching degree of a rule $r$ with a new object $x$ can be defined as formula (4) $\operatorname{matching}(x, r)=\sum_{a \in S_{-} C A(r) \wedge v_{a}=v_{a}^{\prime}} \operatorname{sig}(a)$

Where $V_{a}$ and $V_{a}$ are values of condition attribute $a$ in rule $r$ and attribute $a$ in the new object $x$ respectively.

Definition6 the partial matching set of the new object $x$ denoted $\operatorname{PRUL}(x, \varepsilon)$ will be defined as formula (5):

$\operatorname{PRUL}(x, \varepsilon)=\{r \mid$ matching $(x, r) \geq \varepsilon\}$

where $\varepsilon$ is a given value, and $0<\varepsilon<1$.

Proposition 3 The partial matching set of the new object $x$ can be classified three cases.

(1) $\operatorname{PRUL}(x, \varepsilon)=\Phi$.

(2) $\operatorname{PRUL}(x, \varepsilon) \neq \Phi$, values of rules' decision attributes in $\operatorname{PRUL}(x, \varepsilon)$ are unique.

(3) $\operatorname{PRUL}(x, \varepsilon) \neq \Phi$, values of rules' decision attributes in $\operatorname{PRUL}(x, \varepsilon)$ are not unique.

According to above definitions, the algorithm of uncertain reasoning considering subjective factors when the new object does not match any decision rules will be presented as follows:

Input: the minimum set of decision rules $M$; a new object $X$; the given value of $\varepsilon, 0<\varepsilon<1$.

Output: the result of the reasoning.

Step1: Compute $P R U L(x, \varepsilon)$ by formula (5);

Step2: If $\operatorname{PRUL}(x, \varepsilon)=\Phi$, decrease the value of $\varepsilon$, and return to step1, else go to step 3; 
Step3: If conclusions of rules in $\operatorname{PRUL}(x, \varepsilon)$ are unique, the unique conclusion is the result of reasoning, else go to step4;

Step4: Computer the fit degree of every conclusion in the partial matching set of the new object $x$ by formula (6)

$$
\text { fitness }(x, \beta)=\bigcup_{\alpha \rightarrow \beta \in P R U L(x)} \sum_{a \in S_{-} C A(\alpha \rightarrow \beta)} \operatorname{sig}(a)
$$

Step5: Standardize fit degrees by formula (2);

Step6: compute the general weight by formula (3). The conclusion whose the value of weight $(x, \beta)$ is maximum is the result of reasoning.

Under these circumstances, the accuracy of the algorithm is low, and decisions involve risk, especially when the value of $\varepsilon$ is smaller. Human judgments and preferences must be used.

\section{Conclusion}

In this paper, the algorithm of decision making in this intelligent decision support system is discussed in detail. In order to make decision, the minimum set of decision rules should be computed by simplification of decision table firstly, and the result of decision can be obtained by comparing the new object and the minimum set of decision rules. The novel approach given in this paper can better reflect the needs of users, because both subjective factors representing the users' preferences for various attributes and objective factors such as support degree of decision rules are taken into account in the process of decision making, and the proportion of subjective factors and objective factors can be adjusted according to the actual needs of the users by the ratio of subjective factors. The algorithm considering both subjective factors and objective factor is better than the method based on the support degree of decision rule in flexibility and practicability. But there are some shortcomings, for example, there are two restrictive conditions in the process of making decision: $S_{-} C A(x) \supseteq \bigcup_{r \in R U L} S_{-} C A(r)$ and $0<\operatorname{sig}(a)<1$, and the accuracy of this algorithm is low and decisions involve risk when the new object does not match any decision rules. The method must be researched forward.

\section{References}

[1] RH Bonczek, CW Holsapple, AB Whinston. Founddations of decision support systems, Academic Press, 2014.

[2] Phillips-Wren G. Intelligent decision support systems. Multicriteria Decision Aid and Artificial Intelligence,2013(18) 25-44.

[3] Kantardzic M. Data mining: concepts, models, methods, and algorithms, John Wiley \& Sons, 2011.

[4] Larose D T. Discovering knowledge in data: an introduction to data mining, John Wiley \& Sons, 2014.

[5] Pawlak Z. Rough sets. International Journal of Computer \& Information Sciences, 1982(11) 341-356.

[6] Polkowski L. Rough sets: Mathematical foundations, Springer Science \& Business Media, 2013.

[7] Tian Y, Wang Y, Gavrilova M L. A formal knowledge representation system (FKRS) for the intelligent knowledge base of a cognitive learning engine. International Journal of Software Science and Computational Intelligence (IJSSCI), 2011(3) 1-17.

[8] Pawlak Z, Skowron A. Rudiments of rough sets. Information sciences, 2007(177) 3-27. 